\title{
Los factores estimuladores de colonias reducen la estadía hospitalaria de neutropénicos febriles
}

\author{
Objetivo \\ Evaluar la seguridad y la efectividad de añadir factores estimu- \\ ladores de colonias (FEC) a los antibióticos (ATB) en el tratamien- \\ to de la neutropenia febril causada por quimioterapia del cáncer.
}

Fuente de Datos y Selección de Estudios

Principales bases de datos electrónicas: CANCERLIT, EMBASE, LILACS, MEDLINE, SCI y el Cochrane Controlled Trials Register. Se consultó a los expertos y se examinaron las referencias de los artículos pertinentes. Se seleccionaron ensayos controlados aleatorizados (ECA) que compararan FEC más antibióticos vs. sólo antibióticos para el tratamiento de la neutropenia febril.

\section{Extracción de datos}

Dos revisores de forma independiente, hicieron la selección, la evaluación crítica y la extracción de los datos de los estudios.

\section{Resultados Principales}

Se rastrearon más de 8000 referencias incluyéndose 13 ECA. La mortalidad global no se vio influenciada por el uso de los FEC. Se obtuvo significancia limítrofe en la reducción de la mortalidad asociada a infección, pero este resultado fue muy influenciado por un estudio y desapareció al ser excluido éste. El poder para hallar reducciones de mortalidad global y asociada a infección fue de 33 y $66 \%$ respectivamente, lo que no permite descartar reducciones clínicamente significativas.

El tratamiento con FEC se asoció a un período más corto de hospitalización y de recuperación neutrofílica (Tabla).

Tabla. Resultados del meta-análisis Factores Estimulantes de Colonia vs controles en neutropénicos febriles

\begin{tabular}{l|c|c|c|c}
\multicolumn{1}{c|}{ Resultados } & $\begin{array}{c}\text { FEC + ATB } \\
\text { N/N (\%) }\end{array}$ & $\begin{array}{c}\text { ATB } \\
\text { n/N (\%) }\end{array}$ & $\begin{array}{c}\text { OR/AR } \\
\text { (IC 95\%) }\end{array}$ & p \\
\hline Mortalidad & $34 / 670(5,1)$ & $45 / 633(7,1)$ & OR $0,68(0,43-1,08)$ & 0,1 \\
\hline Mortalidad por infección & $14 / 459(3,1)$ & $24 / 419(5,7)$ & OR $0,51(0,26-1,00)$ & 0,05 \\
\hline Estadía hospitalaria & $144 / 630(22,9)$ & $189 / 591(32,0)$ & HR $0,63(0,49-0,82)$ & 0,0006 \\
\hline Tiempo de recuperación neutrofilica & $105 / 419(25,1)$ & $170 / 375(45,3)$ & HR $0,32(0,23-0,46)$ & $<0.00001$ \\
\hline Algias/síntomas pseudogripales & $47 / 328(14,3)$ & $25 / 294(8,5)$ & OR $2,05(1,22-3,46)$ & 0.007 \\
\hline
\end{tabular}

\section{Conclusiones}

El uso de FEC en pacientes con neutropenia febril por quimioterapia oncológica no afecta la mortalidad global, pero reduce la estadía hospitalaria y el período de recuperación de neutrófilos. No está claro si influencian la mortalidad relacionada con la infección.

Fuente de Financiamiento: H Lee Moffitt Cancer Center USA

\section{Comentario}

La neutropenia febril es frecuente y potencialmente mortal en los pacientes con cáncer sometidos a quimioterapia. ${ }^{1}$ El tratamiento usual es de soporte y antibióticos. Los FEC estimulan y aceleran la producción de una o más líneas celulares en la médula ósea. Sí se detectó un efecto significativo de los FEC sobre la estadía hospitalaria y dicho efecto se mantuvo luego de varios análisis de subgrupos y de sensibilidad. Este beneficio, tiene el potencial de cambiar la práctica clínica actual pues una reducción de la duración de la hospitalización implicaría menores costos, pero se debería realizar un análisis económico que considere el costo de los FEC. Esta duración más corta en el hospital también podría representar una mejor calidad de vida para los pacientes ${ }^{2},{ }^{3}$ pero no se realizó un análisis formal de la calidad de vida.

El efecto de los FEC sobre el tiempo para la recuperación de neutrófilos era esperable, pero es la primera vez que se relaciona esta recuperación más rápida con un beneficio clínico, como una menor estadía hospitalaria.
Los efectos secundarios de los FEC tales como el dolor óseo, artralgias y los síntomas pseudogripales fueron frecuentes y en algunos informes intensos, pero no potencialmente mortales. Estos efectos secundarios se informaron con más frecuencia en el grupo de GM-FEC.

\section{Conclusión del comentador}

El uso de FEC en pacientes neutropénicos febriles por quimioterapia no cambia la mortalidad pero reduce el tiempo de internación y el tiempo para la recuperación neutrofílica. Excepto en pacientes de bajo riesgo, es poco probable que los ECA futuros presenten un grupo control sin tratamiento. Por consiguiente, se debería realizar un meta-análisis de datos de pacientes individuales para evaluar el efecto de los FEC sobre la mortalidad.

Agustín Ciapponi [ Unidad de Medicina Familiar, Hospital Italiano de Buenos Aires. ]

Ciapponi A. Los factores estimuladores de colonias reducen la estadía hospitalaria de neutropénicos febriles. Evid actual pract ambul $2005 ; 8: 163$. Comentado de: Clark OAC, Lyman G, Castro AA, Clark LGO, Djulbegovic B. Colony-stimulating factors for chemotherapy-induced febrile neutropenia: a meta-analysis of randomized controlled trials. J Clin Oncol. 2005;23(18):4198-214.

\section{Referencias}

1. Aviles A, Guzman R, Garcia EL, Talavera A, Diaz-Maqueo JC. Results of a randomized trial of granulocyte colony-stimulating factor in patients with infection and severe granulocytopenia. Anticancer Drugs 1996;7(4):392-7.

2. Smeenk FW, van Haastregt JC, de Witte LP, Crebolder HF. Effectiveness of home care programmes for patients with incurable cancer on their quality of life and time spent in hospital: systematic review. BMJ 1998;316(7149):1939-1944.

3. Berghmans T, Paesmans M, Lafitte J J, Mascaux C, Meert A P, Jacquy C, Burniat A, Steels E, Vallot F, Sculier JP. Therapeutic use of granulocyte and granulocyte-macrophage colony-stimulating factors in febrile neutropenic cancer patients. A systematic review of the literature with meta-analysis. Support Care Cancer 2002;10(3):181-188. 